\title{
ARC Fusion Protein SL-279252
}

National Cancer Institute

\section{Source}

National Cancer Institute. ARC Fusion Protein SL-279252. NCI Thesaurus. Code C161803.

An agonist redirected checkpoint (ARC) fusion protein consisting of the extracellular domains of human programmed cell death 1 (PD-1; PDCD1; CD279) and tumor necrosis factor ligand superfamily member 4 (TNFSF4; OX40 ligand; OX40L; CD252), linked by a central Fc domain (PD1-Fc-OX40L), with potential immunostimulatory and antineoplastic activities. Upon intravenous administration, ARC fusion protein SL-279252 simultaneously binds to both tumor necrosis factor receptor superfamily member 4 (TNFRSF4; OX40) and PD-1 expressed on T-lymphocytes. Stimulation of OX40 may promote cytokine production and induce proliferation of memory and effector T-lymphocytes against tumor cells, while PD-1 binding disrupts PD-1 signaling and may restore immune function through the activation of T-cells. This may enhance the immune-mediated elimination of tumor cells more effectively than PD-1 blockade or OX40-agonism alone. OX40L, a cell surface glycoprotein and member of the tumor necrosis factor (TNF) ligand family, provides a co-stimulatory signal for the proliferation and survival of activated T-cells. PD1, a transmembrane protein in the immunoglobulin superfamily (IgSF) expressed on Tcells, functions as an immune checkpoint that negatively regulates $\mathrm{T}$-cell activation and effector function when activated by its ligands programmed cell death-1 ligand 1 (PD-L1; CD274) or 2 (PD-L2); it plays an important role in tumor evasion from host immunity. 